\title{
CB Research Square \\ Better Health Related Quality of Life Reduced Elevated Lp(a) Levels in Man
}

Jinlan Bao

Sun Yat-Sen Memorial Hospital

ZhuZhi Wen

Sun Yat-Sen University 2nd Affiliated Hospital: Sun Yat-Sen Memorial Hospital

Ying Tang

Sun Yat-Sen University 2nd Affiliated Hospital: Sun Yat-Sen Memorial Hospital

Runlu Sun

Sun Yat-Sen University 2nd Affiliated Hospital: Sun Yat-Sen Memorial Hospital

Canxia Huang

Sun Yat-Sen University 2nd Affiliated Hospital: Sun Yat-Sen Memorial Hospital

JingFeng Wang

Sun Yat-Sen University 2nd Affiliated Hospital: Sun Yat-Sen Memorial Hospital

Yuling Zhang ( $\square$ zzhangyuling@126.com )

Sun Yat-Sen Memorial Hospital https://orcid.org/0000-0002-0292-1271

Research

Keywords: Health related quality of life, Elevated Lipoprotein(a) levels, Premature coronary heart disease

Posted Date: September 2nd, 2020

DOI: https://doi.org/10.21203/rs.3.rs-67835/v1

License: (9) This work is licensed under a Creative Commons Attribution 4.0 International License. Read Full License 


\section{Abstract}

Background: Elevated Lipoprotein(a) $(L p(a))$ is an independent risk factor for premature coronary heart disease (PCHD), but drug therapy rarely changes. Recently, health related quality of life (HRQOL) has been used to measure the prognosis of PCHD, but it is unclear whether HRQOL can improve Lp(a) levels.

Aim: To explore whether HRQOL score in PCHD patients is closely related to increased Lp(a) level.

Methods: The retrospective study included 448 male patients, 218 male patients with PCHD (49.63 \pm 3.72 years) and 230 male healthy controls, the two groups were matched in age. According to $L p(a)$ levels, they were divided into normal $L p(a)$ group $(L p(a)$ $<30 \mathrm{mg} / \mathrm{dL}$ ) and elevated $\mathrm{Lp}(\mathrm{a})$ group $(\mathrm{Lp}(\mathrm{a}) \geq 30 \mathrm{mg} / \mathrm{dL})$. All participants received HRQOL scores. HRQOL scores were assessed using the health Survey of 36 items (SF-36).

Results: Elevated Lp(a) subgroup had more PCHD patients 150(65.5\%) than normal Lp(a) subgroup 68(31.1\%), P<0.001. In elevated $L p(a)$ subgroup and normal $L p(a)$ subgroup, the $L p(a)$ level of PCHD patients were significantly higher than health healthy controls, and HRQOL scores were significantly lower than healthy controls $(P<0.001)$. HRQOL scores significantly reduced the risk of $P C H D$ $(P<0.001)$, while $L p(a)$ levels significantly increased the risk of PCHD $(P<0.001)$. HRQOL scores in the whole $L p(a)$ group were negatively correlated with $L p(a)$ levels $(r=-0.131, P=0.006)$, but not with the elevated $L p(a)$ and normal $L p(a)$ subgroups.

Conclusion: Better HRQOL may be an important indicator to reduce the risk of high Lp(a) and PCHD in man.

\section{Introduction}

Lipoprotein(a) $(L p(a))$ is an independent risk factor for premature coronary heart disease (PCHD $)^{1-5}$.Strong evidence from epidemiological and genome-wide association studies shown that elevated Lp(a) concentration has an important impact in the coronary heart disease progression ${ }^{6}$. Specially, $\mathrm{Lp}(\mathrm{a}) \geq 30 \mathrm{mg} / \mathrm{dL}$ was recommended to be considered as a risk factor for $\mathrm{CHD}$ in the 2016 Canadian cardiovascular meeting ${ }^{7,8}$. When the $\mathrm{Lp}(\mathrm{a})$ concentration lower by $10 \mathrm{mg} / \mathrm{dL}$, the risk of CHD will reduce $5.8 \%$ with the significant level ${ }^{9}$. Similarly, when the Lp(a) concentration lower by $101.5 \mathrm{mg} / \mathrm{dL}$ or the LDL-C will reduce $38.67 \mathrm{mg} / \mathrm{dL}$, the risk of CHD is also significantly reduced ${ }^{5,9}$. However, few drugs are currently effective in reducing Lp(a) levels ${ }^{4,9}$.JUPITER (Justification for the Use of Statins in Prevention: an Intervention Trial Evaluating Rosuvastatin) study reported that statin regulating $L p(a)$ levels as similar as placebo ${ }^{10}$ and highly elevated $L p(a)$ are residual coronary heart disease risk in LDL-C controlled populations ${ }^{10,11}$. A meta-analysis reported that statin can reduce the LDL-C levels by $39 \%$ without changing Lp(a) level ${ }^{12}$. Furthermore, a recent study measured $L p(a)$ levels in 3,896 patients at pre- and post-statin therapy, founding that the mean $L p(a)$ levels was increased $11 \%$ by atorvastatin, pravastatin, rosuvastatin, pitavastatin, and simvastatin/ezetimibe treatment ${ }^{11}$. Currently, neither treatment can effectively reduce the $L p(a)$ level in a PCSK9 Phase II conducted trial ${ }^{13}$.

Health related quality of life(HRQOL) is used progressively as a measure of the outcome of CHD in the previous studies ${ }^{14}$, while HRQOL can effectively improve the Lp(a) levels remain poorly understood. Coronary heart disease has worse HRQOL than healthy controls, and well treated patients have better HRQOL than that of untreated patients ${ }^{15,16}$. EUROASPIRE III survey reported that poor HRQOL can predict morbidity and mortality of CHD patients when standard risk factors remained unchanged ${ }^{17}$. HRQOL was considered as an individual's subjective evaluation of his own health and well-being ${ }^{18}$, which assesses the risk factors of disease and the effectiveness of intervention and therapy ${ }^{15}$. HRQOL has great advantage in measuring special disease ${ }^{14}$, including mental components and physical components, which are measured by using the 36 -Item Short Form Health Survey $(\mathrm{SF}-36)^{15,18,19}$. Moreover, depression, anxiety, and psychotic disorders are independent risk factors for cardiovascular morbidity and mortality 20,21 . In addition, mental disorders are found to increase PCHD risk ${ }^{5,21}$.

This study aims to investigate the relationship between HRQOL and elevated Lp(a) levels in PCHD patients and to explore whether HRQOL is an important measure to significantly reduce $L p(a)$ levels in PCHD patients

\section{Materials And Methods}


This is a retrospective single center observation study, we consecutively enrolled 218 male patients at Department of Cardiology of Sun Yat-sen Memorial Hospital between January 2017 and January 2020. All patients were aged between 18-55 years and had been underwent coronary angiography, they were diagnosed with $\mathrm{PCHD}^{22}$, include 49 ST-segment elevation myocardial infarction (STEMI), 45non-ST-segment elevation myocardial infarction (non-STEMI) and 124 unstable angina patients. There were 230 age matched male health controls. All participants undergo coronary angiography, who had at least one visible stenosis in any major vessel $\geq 50 \%$ as PCHD patients, and major vessel without stenosis was considered as health controls. Meanwhile, rheumatic heart disease, valvular disease, cardiomyopathy, congenital heart disease and severe hepatic and renal insufficiency were excluded from this study.

Clinical characteristics, biochemical indicator, coronary angiography and HRQOL measurements by SF-36 were collected. The study complied with the Declaration of Helsinki and approved by the Ethics Committee of Sun Yat-sen Memorial Hospital of Sun Yat-sen University.

\subsection{HRQOL and SF-36}

HRQOL was measured self-rated health over the past 4 weeks by using the SF36-Item ${ }^{18,23}$.SF-36contained the physical component summary (PCS) and the mental component summary (MCS).PCS contained four domains: physical functioning (10 items), rolephysical limitation (4 items), bodily Pain ( 2 items), general Health (5 items).MCS contained four domains: vitality (4 items), social functioning (2items), role-emotional limitation(3 items), and mental health (5items).For each domain, score ranked from 0 (worst health) to 100 (best health). Total score was ranged from 0 to 100, the mental and physical health scores were combined from eight z-scores by using weights, standardized to mean $50 \pm 10^{19}$ (supplement Table1). 
Table 1

Clinical characteristics for Elevated Lp(a) and normal Lp(a) subgroup

\begin{tabular}{|c|c|c|c|c|c|c|c|c|c|}
\hline & $\begin{array}{l}\text { Total } \\
\text { Lp(a) } \\
\text { group }\end{array}$ & & & $\begin{array}{l}\text { Normal } \\
\text { Lp(a) } \\
\text { subgroup }\end{array}$ & & & $\begin{array}{l}\text { Elevated } \\
\text { Lp(a) } \\
\text { subgroup }\end{array}$ & & \\
\hline & $\begin{array}{l}\text { PCHD } \\
\text { patients } \\
\mathrm{N}=218\end{array}$ & $\begin{array}{l}\text { Health } \\
\text { controls } \\
\mathrm{N}=230\end{array}$ & $\mathrm{P}$ & $\begin{array}{l}\mathrm{PCHD} \\
\text { patients } \\
\mathrm{N}=68\end{array}$ & $\begin{array}{l}\text { Health } \\
\text { controls } \\
\mathrm{N}=151\end{array}$ & $P$ & $\begin{array}{l}\text { PCHD } \\
\text { patients } \\
\mathrm{N}=150\end{array}$ & $\begin{array}{l}\text { Health } \\
\text { controls } \\
\mathrm{N}=79\end{array}$ & $P$ \\
\hline Age, years & $\begin{array}{l}49.63 \pm \\
3.72\end{array}$ & $\begin{array}{l}49.48 \pm \\
4.24\end{array}$ & 0.682 & $\begin{array}{l}49.64 \pm \\
3.35\end{array}$ & $\begin{array}{l}49.05 \pm \\
4.78\end{array}$ & 0.405 & $\begin{array}{l}49.43 \pm \\
3.83\end{array}$ & $\begin{array}{l}49.84 \pm \\
3.52\end{array}$ & 0.434 \\
\hline $\mathrm{BMI}(\mathrm{KG} / \mathrm{m} 2)$ & $\begin{array}{l}23.59 \pm \\
1.29\end{array}$ & $\begin{array}{l}23.31 \pm \\
1.65\end{array}$ & 0.045 & $\begin{array}{l}23.49 \pm \\
1.18\end{array}$ & $\begin{array}{l}23.36 \pm \\
1.73\end{array}$ & 0.574 & $\begin{array}{l}23.63 \pm \\
1.34\end{array}$ & $\begin{array}{l}23.21 \pm \\
1.48\end{array}$ & 0.029 \\
\hline Hypertension, n(\%) & $74(33.9 \%)$ & $65(28.3 \%)$ & 0.220 & $23(33.8 \%)$ & $46(30.7 \%)$ & 0.641 & $51(34 \%)$ & $19(23.8 \%)$ & 0.133 \\
\hline Diabetes, n(\%) & $51(23.4 \%)$ & $35(15.2 \%)$ & 0.031 & $13(19.1 \%)$ & $26(17.3 \%)$ & 0.849 & $38(25.3 \%)$ & $9(11.3 \%)$ & 0.016 \\
\hline $\begin{array}{l}\text { Hyperlipidemia, } \\
\text { n(\%) }\end{array}$ & $21(9.6 \%)$ & $23(10 \%)$ & 1 & $9(13.2 \%)$ & $15(10 \%)$ & 0.490 & $12(8.0 \%)$ & $8(10 \%)$ & 0.628 \\
\hline $\begin{array}{l}\text { Hyperuricemia, } \\
\mathrm{n}(\%)\end{array}$ & $21(9.6 \%)$ & $20(8.7 \%)$ & 0.746 & $8(11.8 \%)$ & $11(7.3 \%)$ & 0.305 & $13(8.7 \%)$ & $9(11.3 \%)$ & 0.638 \\
\hline Smoking, n(\%) & $70(32.1 \%)$ & $49(21.3 \%)$ & 0.010 & $27(39.7 \%)$ & $34(22.7 \%)$ & 0.014 & $43(28.7 \%)$ & $15(18.8 \%)$ & 0.112 \\
\hline Drinking, n(\%) & $14(6.4 \%)$ & $16(7.0 \%)$ & 0.852 & $3(4.4 \%)$ & $11(7.3 \%)$ & 0.557 & $11(7.3 \%)$ & $5(6.3 \%)$ & 1 \\
\hline $\begin{array}{l}\text { PCHD family } \\
\text { history, } \mathrm{n}(\%)\end{array}$ & $17(7.8 \%)$ & $15(6.5 \%)$ & 0.714 & $5(7.4 \%)$ & $11(7.3 \%)$ & 1 & $12(8.0 \%)$ & $4(5 \%)$ & 0.587 \\
\hline $\begin{array}{l}\text { Cholesterol } \\
\text { therapy, n(\%) }\end{array}$ & $22(10.1 \%)$ & $21(9.1 \%)$ & 0.751 & $5(7.4 \%)$ & $14(9.3 \%)$ & 0.797 & $17(11.3 \%)$ & $7(8.8 \%)$ & 0.654 \\
\hline $\mathrm{TC}(\mathrm{mmol} / \mathrm{L})$ & $\begin{array}{l}5.64 \pm \\
1.30\end{array}$ & $\begin{array}{l}4.95 \pm \\
1.19\end{array}$ & $<.001$ & $\begin{array}{l}5.55 \pm \\
1.37\end{array}$ & $\begin{array}{l}4.88 \pm \\
1.20\end{array}$ & $<0.001$ & $\begin{array}{l}5.68 \pm \\
1.27\end{array}$ & $\begin{array}{l}5.08 \pm \\
1.19\end{array}$ & 0.001 \\
\hline $\mathrm{TG}(\mathrm{mmol} / \mathrm{L})$ & $\begin{array}{l}1.67 \pm \\
0.83\end{array}$ & $\begin{array}{l}1.59 \pm \\
0.99\end{array}$ & 0.395 & $\begin{array}{l}1.66 \pm \\
0.75\end{array}$ & $\begin{array}{l}1.67 \pm \\
1.12\end{array}$ & 0.942 & $\begin{array}{l}1.67 \pm \\
0.86\end{array}$ & $\begin{array}{l}1.44 \pm \\
0.65\end{array}$ & 0.043 \\
\hline $\mathrm{HDL}-\mathrm{C}(\mathrm{mmol} / \mathrm{L})$ & $\begin{array}{l}1.33 \pm \\
0.36\end{array}$ & $\begin{array}{l}1.27 \pm \\
0.31\end{array}$ & 0.069 & $\begin{array}{l}1.29 \pm \\
0.31\end{array}$ & $\begin{array}{l}1.24 \pm \\
0.30\end{array}$ & 0.237 & $\begin{array}{l}1.34 \pm \\
0.38\end{array}$ & $\begin{array}{l}1.33 \pm \\
0.33\end{array}$ & 0.753 \\
\hline LDL-C(mmol/L) & $\begin{array}{l}3.58 \pm \\
0.92\end{array}$ & $\begin{array}{l}3.08 \pm \\
0.90\end{array}$ & $<.001$ & $\begin{array}{l}3.54 \pm \\
0.98\end{array}$ & $\begin{array}{l}3.06 \pm \\
0.90\end{array}$ & $<0.001$ & $\begin{array}{l}3.60 \pm \\
0.90\end{array}$ & $\begin{array}{l}3.14 \pm \\
0.91\end{array}$ & $\begin{array}{l}< \\
0.001\end{array}$ \\
\hline apoA1 (g/L) & $\begin{array}{l}1.36 \pm \\
0.30\end{array}$ & $\begin{array}{l}1.28 \pm \\
0.25\end{array}$ & 0.002 & $\begin{array}{l}1.33 \pm \\
0.28\end{array}$ & $\begin{array}{l}1.24 \pm \\
0.23\end{array}$ & 0.009 & $\begin{array}{l}1.37 \pm \\
0.31\end{array}$ & $\begin{array}{l}1.35 \pm \\
0.28\end{array}$ & 0.682 \\
\hline apoB (g/L) & $\begin{array}{l}1.03 \pm \\
0.26\end{array}$ & $\begin{array}{l}0.90 \pm \\
0.25\end{array}$ & $\begin{array}{l}< \\
0.001\end{array}$ & $\begin{array}{l}1.01 \pm \\
0.28\end{array}$ & $\begin{array}{l}0.90 \pm \\
0.25\end{array}$ & 0.004 & $\begin{array}{l}1.04 \pm \\
0.25\end{array}$ & $\begin{array}{l}0.91 \pm \\
0.26\end{array}$ & 0.001 \\
\hline apoE (mg/L) & $\begin{array}{l}37.48 \pm \\
11.59\end{array}$ & $\begin{array}{l}37.44 \pm \\
13.72\end{array}$ & 0.974 & $\begin{array}{l}37.08 \pm \\
13.35\end{array}$ & $\begin{array}{l}37.74 \pm \\
14.88\end{array}$ & 0.755 & $\begin{array}{l}37.67 \pm \\
10.74\end{array}$ & $\begin{array}{l}36.89 \pm \\
11.30\end{array}$ & 0.609 \\
\hline $\mathrm{Lp}(\mathrm{a})(\mathrm{mg} / \mathrm{dL})$ & $\begin{array}{l}50.31 \pm \\
32.81\end{array}$ & $\begin{array}{l}26.42 \pm \\
21.93\end{array}$ & $\begin{array}{l}<.001 \\
0.001\end{array}$ & $\begin{array}{l}17.97 \pm \\
5.91\end{array}$ & $\begin{array}{l}13.08 \pm \\
6.82\end{array}$ & $\begin{array}{l}< \\
0.001\end{array}$ & $\begin{array}{l}64.97 \pm \\
29.29\end{array}$ & $\begin{array}{l}51.43 \pm \\
18.32\end{array}$ & $\begin{array}{l}< \\
0.001\end{array}$ \\
\hline Creatinine(umol/L) & $\begin{array}{l}83.11 \pm \\
24.25\end{array}$ & $\begin{array}{l}83.45 \pm \\
26.92\end{array}$ & 0.886 & $\begin{array}{l}84.81 \pm \\
22.09\end{array}$ & $\begin{array}{l}83.24 \pm \\
27.16\end{array}$ & 0.678 & $\begin{array}{l}82.33 \pm \\
25.20\end{array}$ & $\begin{array}{l}83.85 \pm \\
26.61\end{array}$ & 0.671 \\
\hline Uric Acid(umol/L) & $\begin{array}{l}369.16 \pm \\
100.43\end{array}$ & $\begin{array}{l}379.72 \pm \\
114\end{array}$ & 0.300 & $\begin{array}{l}371.62 \pm \\
106.51\end{array}$ & $\begin{array}{l}372.65 \pm \\
102.08\end{array}$ & 0.945 & $\begin{array}{l}368.04 \pm \\
97.90\end{array}$ & $\begin{array}{l}392.96 \pm \\
133.21\end{array}$ & 0.108 \\
\hline
\end{tabular}
$E(A p o E) ; p$-value $<0.05$ was considered statistically significant. 


\begin{tabular}{|c|c|c|c|c|c|c|c|c|c|}
\hline \multirow[b]{2}{*}{$\mathrm{FPG}(\mathrm{mmol} / \mathrm{L})$} & \multicolumn{3}{|l|}{$\begin{array}{l}\text { Total } \\
\text { Lp(a) } \\
\text { group }\end{array}$} & \multicolumn{3}{|c|}{$\begin{array}{l}\text { Normal } \\
\text { Lp(a) } \\
\text { subgroup }\end{array}$} & \multicolumn{3}{|c|}{$\begin{array}{l}\text { Elevated } \\
\text { Lp(a) } \\
\text { subgroup }\end{array}$} \\
\hline & $\begin{array}{l}6.01 \pm \\
1.59\end{array}$ & $\begin{array}{l}5.57 \pm \\
1.34\end{array}$ & 0.001 & $\begin{array}{l}5.96 \pm \\
1.59\end{array}$ & $\begin{array}{l}5.65 \pm \\
1.45\end{array}$ & 0.161 & $\begin{array}{l}6.03 \pm \\
1.59\end{array}$ & $\begin{array}{l}5.40 \pm \\
1.09\end{array}$ & 0.002 \\
\hline
\end{tabular}

2.3 Measurements of LP(a) levels and Coronary angiography

Blood samples were collected fast for 8 hours. Measurements of lipoprotein(a) were used particle enhanced quantitative turbidimetric immunoassay (PETIA) (Tina-quant ${ }^{\circledR}$ Lipoprotein (a) Gen.2, Roche Diagnostics International Ltd.), on a COBAS automated chemistry analyzer. In this study, $L p(a) \geq 30 \mathrm{mg} / \mathrm{dL}$ was considered as elevated ${ }^{7,8}$ and $\mathrm{Lp}(\mathrm{a})<30 \mathrm{mg} / \mathrm{dL}$ was considered as normal. $L p(a)$ was divided into two subgroups according to it level: normal $L p(a)$ subgroup and elevated $L p(a)$ subgroup.

2.4 Diagnosis of hypertension, hyperlipidemia and other factors

Hypertension was defined as blood pressure $\geq 140 / 90 \mathrm{mmHg}$ and/or took antihypertensive drug therapy previously. Hyperlipidemia was defined as total cholesterol $(T C) \geq 5.18 \mathrm{mmol} / \mathrm{L}$; low-density lipoprotein cholesterol $(\mathrm{LDL}-\mathrm{C})>3.37 \mathrm{mmol} / \mathrm{L}$ and/or lipid lowering therapy; Diabetes mellitus (DM)were defined as FPG $\geq 7.0 \mathrm{mmol} / \mathrm{L}$ and 2-hour post-meal plasma glucose $\geq 11.1 \mathrm{mmol} / \mathrm{L}$, and/or glucose lowering treatment. Body mass index (BMI) was defined as the body weight $(\mathrm{kg})$ divided by the square of the height $(\mathrm{m} 2)$ and $\mathrm{BMI}>28.0 \mathrm{~kg} / \mathrm{m} 2$ was defined as obesity. Smokers were defined by at least one cigarette per day. Drinking were defined as at least $50 \mathrm{~g}$ wins per day.

\subsection{Statistical analysis}

Continuous variables are presented as mean \pm standard deviation or median and interquartile range (IQR), the independent-samples T-test or Mann-Whitney test was used to compare two continuous variable groups. One-way analysis of variance was used for comparison among multiple groups. Categorical variables are presented as numbers and percentages $\mathrm{n}(\%)$, using Pearson chisquare test. Multiple linear regression model was used to investigate the relationship between $L p(a)$ and HRQOL score. Multivariate logistic regression model was used to determine association between $L p(a)$ levels and PCHD, adjusted for diabetes, smoking, TC, LDL-C, ApoA1, ApoB, FBG and BMI. p-value $<0.05$ was considered statistically significant. SPSS 23.0 statistical software version was used to all statistical analyses.

\section{Results}

\subsection{Clinical and biochemical characteristics}

Elevated Lp(a) subgroup had more PCHD patients than normal Lp(a) subgroup 150(65.5\%) VS 68(31.1\%), P<0.001.In normal Lp(a) subgroup, PCHD patients had higher smokers than health controls $27(39.7 \%)$ VS $34(22.7 \%), p=0.014$, but no different in elevated Lp(a) subgroup. In elevated Lp(a) subgroups and normal Lp(a) subgroups, PCHD patients showed higher Lp(a), TC, LDL-C, ApoB levels than health controls (Table 1).

\subsection{HRQOL and SF-36}

In elevated $L p(a)$ and normal $L p(a)$ subgroup, PCHD patients significantly lower HRQOL, mental health, vitality, role-emotional limitation and role-physical limitation than health controls (Table 2). Health controls were significantly high HRQOL than STEMI, non-STEMI and unstable angina patients, no different in three groups (Fig. 1). 
Table 2

HRQOL and SF-36

\begin{tabular}{|c|c|c|c|c|c|c|c|c|c|}
\hline & $\begin{array}{l}\text { Total Lp(a) } \\
\text { group }\end{array}$ & & & $\begin{array}{l}\text { Normal } \\
\text { Lp(a) } \\
\text { subgroup }\end{array}$ & & & $\begin{array}{l}\text { Elevated } \\
\text { Lp(a) } \\
\text { subgroup }\end{array}$ & & \\
\hline & $\begin{array}{l}\mathrm{PCHD} \\
\text { patients } \mathrm{N}= \\
218\end{array}$ & $\begin{array}{l}\text { Health } \\
\text { controls } \mathrm{N}= \\
230\end{array}$ & $P$ & $\begin{array}{l}\mathrm{PCHD} \\
\text { patients } \\
\mathrm{N}=68\end{array}$ & $\begin{array}{l}\text { Health } \\
\text { controls } \mathrm{N} \\
=151\end{array}$ & $P$ & $\begin{array}{l}\text { PCHD } \\
\text { patients } \mathrm{N} \\
=150\end{array}$ & $\begin{array}{l}\text { Health } \\
\text { controls } \\
\mathrm{N}=79\end{array}$ & $P$ \\
\hline Vitality & $9.61 \pm 1.27$ & $10.15 \pm 1.12$ & $\begin{array}{l}< \\
0.001\end{array}$ & $\begin{array}{l}9.59 \pm \\
1.19\end{array}$ & $\begin{array}{l}10.21 \pm \\
1.11\end{array}$ & $\begin{array}{l}< \\
0.001\end{array}$ & $9.62 \pm 1.31$ & $\begin{array}{l}10.04 \pm \\
1.12\end{array}$ & 0.016 \\
\hline $\begin{array}{l}\text { Social } \\
\text { functioning }\end{array}$ & $9.95 \pm 1.32$ & $10.22 \pm 1.19$ & 0.024 & $\begin{array}{l}9.98 \pm \\
1.32\end{array}$ & $\begin{array}{l}10.26 \pm \\
1.23\end{array}$ & 0.121 & $9.94 \pm 1.32$ & $\begin{array}{l}10.14 \pm \\
1.11\end{array}$ & 0.251 \\
\hline $\begin{array}{l}\text { Role- } \\
\text { emotional } \\
\text { limitation }\end{array}$ & $7.70 \pm 2.23$ & $9.17 \pm 2.68$ & $\begin{array}{l}< \\
0.001\end{array}$ & $\begin{array}{l}7.66 \pm \\
1.98\end{array}$ & $9.36 \pm 2.55$ & $\begin{array}{l}< \\
0.001\end{array}$ & $7.72 \pm 2.33$ & $\begin{array}{l}8.80 \pm \\
2.89\end{array}$ & 0.002 \\
\hline Mental health & $9.16 \pm 1.26$ & $10.32 \pm 1.07$ & $\begin{array}{l}< \\
0.001\end{array}$ & $\begin{array}{l}9.14 \pm \\
1.17\end{array}$ & $\begin{array}{l}10.40 \pm \\
1.16\end{array}$ & $\begin{array}{l}< \\
0.001\end{array}$ & $9.17 \pm 1.30$ & $\begin{array}{l}10.16 \pm \\
0.86\end{array}$ & $\begin{array}{l}< \\
0.001\end{array}$ \\
\hline $\begin{array}{l}\text { Physical } \\
\text { functioning }\end{array}$ & $10.07 \pm 0.96$ & $10.31 \pm 0.97$ & 0.009 & $\begin{array}{l}10.14 \pm \\
0.97\end{array}$ & $\begin{array}{l}10.35 \pm \\
1.00\end{array}$ & 0.154 & $\begin{array}{l}10.04 \pm \\
0.95\end{array}$ & $\begin{array}{l}10.25 \pm \\
0.90\end{array}$ & 0.109 \\
\hline $\begin{array}{l}\text { Role-physical } \\
\text { limitation }\end{array}$ & $8.39 \pm 2.37$ & $9.0 \pm 1.81$ & 0.002 & $\begin{array}{l}8.32 \pm \\
2.33\end{array}$ & $8.96 \pm 1.90$ & 0.033 & $8.42 \pm 2.40$ & $\begin{array}{l}9.07 \pm \\
1.62\end{array}$ & 0.032 \\
\hline Body pain & $9.89 \pm 1.16$ & $10.01 \pm 1.12$ & 0.266 & $\begin{array}{l}9.80 \pm \\
1.03\end{array}$ & $10 \pm 1.17$ & 0.221 & $9.93 \pm 1.21$ & $\begin{array}{l}10.02 \pm \\
1.04\end{array}$ & 0.572 \\
\hline $\begin{array}{l}\text { General } \\
\text { health }\end{array}$ & $9.87 \pm 1.27$ & $10.32 \pm 1.12$ & $\begin{array}{l}< \\
0.001\end{array}$ & $\begin{array}{l}9.77 \pm \\
1.22\end{array}$ & $\begin{array}{l}10.36 \pm \\
1.15\end{array}$ & 0.001 & $9.91 \pm 1.29$ & $\begin{array}{l}10.23 \pm \\
1.05\end{array}$ & 0.053 \\
\hline HRQOL & $74.64 \pm 5.68$ & $79.49 \pm 6.18$ & $\begin{array}{l}< \\
0.001\end{array}$ & $\begin{array}{l}74.40 \pm \\
4.61\end{array}$ & $\begin{array}{l}79.90 \pm \\
6.19\end{array}$ & $\begin{array}{l}< \\
0.001\end{array}$ & $\begin{array}{l}74.74 \pm \\
6.12\end{array}$ & $\begin{array}{l}78.71 \pm \\
6.13\end{array}$ & $\begin{array}{l}< \\
0.001\end{array}$ \\
\hline
\end{tabular}

3.3Relationship between $L p(a)$ levels and PCHD risk

In elevated $L p(a)$ and normal $L p(a)$ subgroup, $L p(a)$ levels was significantly increase PCHD risk, but HRQOL significantly decrease PCHD risk. Smoking was significantly increase PCHD risk in elevated $L p(a)$ subgroup, but no correlation with normal Lp(a) subgroup (Table 3). 
Table 3

Multiple logistic analysis of relationship between $L p(a)$ level, HRQOL and PCHD risk

\begin{tabular}{|lllllll|}
\hline & Total Lp(a) group & & Normal Lp(a) subgroup & & \multicolumn{2}{l}{ Elevated Lp(a) subgroup } \\
\hline & OR,95\%Cl & $\mathbf{p}$ & OR,95\%Cl & p & OR,95\%Cl & p \\
\hline TC (mmol/L) & $0.830(0.274,2.508)$ & 0.741 & $0.550(0.062,4.853)$ & 0.591 & $1.122(0.222,5.669)$ & 0.889 \\
\hline LDL-C (mmol/L) & $3.224(0.598,17.382)$ & 0.173 & $5.427(0.220,133.731)$ & 0.301 & $2.263(0.187,27.326)$ & 0.521 \\
\hline ApoA1 (g/L) & $1.457(0.377,5.628)$ & 0.585 & $3.710(0.305,45.162)$ & 0.304 & $0.677(0.098,4.696)$ & 0.693 \\
\hline ApoB (g/L) & $0.195(0.012,3.163)$ & 0.250 & $0.137(0.004,5.165)$ & 0.283 & $0.256(0.002,31.400)$ & 0.579 \\
\hline FPG (mmol/L) & $1.297(1.042,1.614)$ & 0.020 & $1.285(0.937,1.762)$ & 0.120 & $1.411(0.994,2.004)$ & 0.054 \\
\hline Diabetes & $0.745(0.335,1.660)$ & 0.472 & $0.465(0.124,1.740)$ & 0.256 & $1.066(0.347,3.276)$ & 0.912 \\
\hline Smoking & $2.150(1.280,3.611)$ & 0.004 & $1.953(0.928,4.113)$ & 0.078 & $2.214(1.034,4.742)$ & 0.041 \\
\hline BMI (KG/m2) & $1.076(0.913,1.267)$ & 0.377 & $1.095(0.858,1.398)$ & 0.464 & $1.116(0.879,1.416)$ & 0.367 \\
\hline Lp(a) (mg/dL & $1.034(1.024,1.044)$ & $<0.001$ & $1.114(1.056,1.175)$ & $<0.001$ & $1.025(1.010,1.041)$ & 0.001 \\
\hline HRQOL & $0.875(0.840,0.913)$ & $<0.001$ & $0.853(0.799,0.912)$ & $<0.001$ & $0.898(0.849,0.950)$ & $<0.001$ \\
\hline Fully adjusted for: TC, LDL-C, apoA1, apoB, FPG, BMI levels, diabetes and smoking history; OR: Odds Ratio:95\%Cl:95\% & & & & \\
\hline Confidence Interval. & & & & & \\
\hline
\end{tabular}

3.4Relationship between Lp(a) level and HRQOL

HRQOL was negatively correlation with $L p(a)$ levels in total $L p(a)$ group $(r=-0.131, P=0.006)$, no correlation with elevated $L p(a)$ levels and normal $L p(a)$ levels. However, mental health was negatively correlated with $L p(a)$ levels $(r=-0.153, p=0.023)$ and rolephysical limitation was positively correlated with $L p(a)$ levels $(r=0.171, p=0.011)$ in elevated $L p(a)$ levels $($ Table 4$)$.

Table 4

Multiple linear regression model for relationship between $\mathrm{LP}(\mathrm{a})$ levels and HRQOL

\begin{tabular}{|c|c|c|c|c|c|c|c|c|c|}
\hline & $\begin{array}{l}\text { Total Lp(a) } \\
\text { group }\end{array}$ & & & $\begin{array}{l}\text { Normal Lp(a) } \\
\text { subgroup }\end{array}$ & & & $\begin{array}{l}\text { Elevated } \\
\text { Lp(a) } \\
\text { subgroup }\end{array}$ & & \\
\hline & $r$ & $\mathrm{t}$ & $\mathrm{p}$ & $r$ & $\mathrm{t}$ & $\mathrm{p}$ & $r$ & $\mathrm{t}$ & $\mathrm{p}$ \\
\hline Vitality & -0.109 & -2.283 & 0.023 & -0.054 & -0.764 & 0.446 & -0.093 & -1.372 & 0.171 \\
\hline Social functioning & -0.095 & -2.028 & 0.043 & 0.069 & 0.990 & 0.323 & -0.121 & -1.814 & 0.071 \\
\hline $\begin{array}{l}\text { Role-emotional } \\
\text { limitation }\end{array}$ & -0.119 & -2.552 & 0.011 & -0.043 & -0.625 & 0.533 & -0.043 & -0.638 & 0.524 \\
\hline Mental health & -0.206 & -4.405 & $<.001$ & -0.077 & -1.066 & 0.288 & -0.153 & -2.287 & 0.023 \\
\hline $\begin{array}{l}\text { Physical } \\
\text { functioning }\end{array}$ & -0.052 & -1.127 & 0.260 & -0.009 & -0.134 & 0.893 & 0.018 & 0.262 & 0.794 \\
\hline $\begin{array}{l}\text { Role-physical } \\
\text { limitation }\end{array}$ & 0.056 & 1.179 & 0.239 & -0.118 & -1.701 & 0.090 & 0.171 & 2.562 & 0.011 \\
\hline Body pain & -0.011 & -0.245 & 0.807 & -0.085 & -1.229 & 0.221 & 0.002 & 0.029 & 0.977 \\
\hline General health & -0.045 & -0.951 & 0.342 & -0.009 & -0.129 & 0.897 & -0.013 & -0.197 & 0.844 \\
\hline HRQOL & -0.131 & -2.762 & 0.006 & -0.088 & -1.246 & 0.214 & -0.031 & -0.459 & 0.647 \\
\hline
\end{tabular}




\section{Discussion}

Previous epidemiological studies have reported that elevated $L p(a)$ levels were the remarkable independent risk factor for premature coronary heart disease (PCHD $)^{1-5}$.The PROCAM Study reported that $L p(a)$ as a risk factor for premature myocardial infarction, and the age of premature events was less than 45 years $^{3,4}$. The GRIP Study reported that evaluated $L p(a)$ levels substantially increases cardiovascular risks in men aged 40-59 years ${ }^{4}$. However,rare available measures were used to reduced $L p(a)$ levels effectively. In this retrospective study, we indicated that PCHD patients showed higher $L p(a)$ levels than health controls, and the $L p(a)$ levels increased PCHD risk remarkably, the results was similar with previously study ${ }^{1-5}$.

Nevertheless, there was no standardized $L p(a)$ measurement in terms of heterogeneity of $L p(a)$ particle sizes ${ }^{9,24,25}$. Several epidemiological, Mendelian randomization and genome-wide association studies reported that elevated $\mathrm{Lp}(\mathrm{a})(\geq 50 \mathrm{mg} / \mathrm{dl})$ is an independent and significant risk factor for $\mathrm{CHD}^{26-28}$. However, this recommendation neglects patients with $\mathrm{Lp}(\mathrm{a})$ level 25$50 \mathrm{mg} / \mathrm{dl}^{2}$. The risk of Calcificaortic valve stenosis (CAVS) was defined as high $\mathrm{Lp}(\mathrm{a})>40$ to $60 \mathrm{mg} / \mathrm{dl}^{2}$. The German and U.K. apheresis guidelines recommended $L p(a)>60 \mathrm{mg} / \mathrm{dl}$ to specially treat who has elevated $L p(a)$ level and recurrent CVD events, or uncontrollable elevated LDL-C ${ }^{11}$.A large meta-analysis collected 126,634 participants and 1.3 million person-years of follow-up, showing that $\mathrm{Lp}(\mathrm{a})>30 \mathrm{mg} / \mathrm{dl}$ increased myocardial infarction risk ${ }^{1}$. The 2016 Canadian Cardiovascular Society Guidelines for the Management of Dyslipidemia recommended $L p(a) \geq 30 \mathrm{mg} / \mathrm{dl}$ as a risk factor for $\mathrm{CHD}^{7,8}$.In our study, we defined $\mathrm{Lp}(\mathrm{a}) \geq 30 \mathrm{mg} / \mathrm{dl}$ as elevated $L p(a)$. Elevated $L p(a)$ subgroup had more PCHD patients than normal $L p(a)$ subgroup, which was consistent with the results of previous research.

Diet, exercise and the majority of lipid lowering drugs rarely change $L p(a)$ levels ${ }^{4}$. Of note, a meta-analysis reported that statin reduced LDL-C levels by $39 \%$ without changing Lp(a) levels ${ }^{12}$. In a PCSK9 Phase II conducted trial, the Lp(a) did not substantially reduced in any dosing groups ${ }^{13} .10 \mathrm{mg}$ Ezetimibe remarkably reduced plasma $\mathrm{Lp}(\mathrm{a})$ levels when compared with placebo ${ }^{29}$. In amipomersen Phase III trials, the mean percent $L p(a)$ levels decreased at 28 weeks was substantially more than placebo group, but it had more side effects, such as injection site reactions, hepatic steatosis and hepatic enzyme elevation ${ }^{9}$. Research reported that CETP inhibitors reduced the mean Lp(a) levels by $25-40 \%$ in a larger Phase II or Phase III clinical trial ${ }^{9,30,31}$. Other studies showed $\mathrm{Lp}(\mathrm{a})$ levels association with daily intake of vitamin D or monounsaturated fatty acids, but no correlation with lifestyle ${ }^{9}$. Smoking cessation was shown to reduce or increase Lp(a) concentrations ${ }^{32}$. Recent genome-wide association studies showed that LPA SNP rs 10455872 was significantly associated with elevated $L p(a)$ levels ${ }^{11}$. However, some individuals have alleles that not express elevated $L p(a)^{11,32}$.Therefore, several therapies have been shown to reduce $L p(a)$ levels, but none of them was widely used clinically 9 .

Currently, HRQOL can effectively improve the elevated $L p(a)$ levels was hardly known. Coronary heart disease has poorer HRQOL than that of healthy controls ${ }^{15,16}$. It was also found that mental disorders increased PCHD risk ${ }^{5,21}$.Psychological interventions had effect on mortality and cardiovascular morbidity ${ }^{33,34}$, while HRQOL can notably decrease Lp(a) levels is still unknown. Our study discovered that mental health can significantly decrease $L p(a)$ levels and PCHD risk in elevated Lp(a) subgroup, while was not correlated with normal elevated $\mathrm{Lp}(\mathrm{a})$ subgroup. Therefore, we suggested that better mental health is a promising protective factor for elevated $L p(a)$ levels. Previously, the association between BMI and $L p(a)$ levels is controversial ${ }^{35}$. BMI was not correlated with $L p(a)$ levels in the Women's Health Study ${ }^{36}$, while it was correlated with 20-29 years young adults in the Cherokee Diabetes Study ${ }^{37}$ and obese South Indian men ${ }^{35,38}$. Our finding suggested that PCHD patients significantly high BMI than health controls in elevated $L p(a)$ group. Moreover, Exercise does not significantly reduce $L p(a)$ levels ${ }^{4}, 9$.Our study indicated that physical functioning and rolephysical limitation did not effectively decrease $L p(a)$. Herein, we suggested that $L p(a)$ levels was not able to be lower by only doing exercise. In our study, PCHD patients high ApoA1 levels than health controls in total Lp(a)group. Some studies reported that $L p(a)$ is made up of apoA covalently bound to ApoB-1009, 11 . IONIS-Apo(a)Rx antisense oligonucleotide selectively reduced the synthesis of apoA in the liver and consequently the plasma concentration of $L p(a)$ in Phase I study ${ }^{9}$. We suggested that decreased apoA levels were another novel therapy for elevated $L p(a)$ levels.

Other risk factors, such as smoking, hypertension, diabetes and dyslipidemia were the independent risk factors for PCHD ${ }^{1,2,4,5}$, our finding was consistent with previous studies. A German's study reported that $61 \%$ current smokers, approximately $40 \%$ active

Page $8 / 13$ 
smokers in young and mid-aged German adults, increases premature MI significantly. In the DEGS1 survey, $40 \%$ of young and $16 \%$ mid-aged German population have hypertension, while other studies showed that the proportion of arterial hypertension was $70 \%$ in German ${ }^{4}$. Hypertriglyceridemia was the most important risk factor for young myocardial infarction ${ }^{39}$. However, triglycerides did not increase PCHD risk in our study, the result was consistent with previous research ${ }^{4}$, and uric acid had no difference between PCHD group and health control group, which is consistent with before ${ }^{40}$, we considered different regions and eating habits.

\section{Limitations}

This data was a selection from China in a single center. First, this study was conducted in a single with limited data, the results of this study was not able to represent general population and other ethnicities. Second, as it was a retrospective study, it would not have follow-up and prospective studies, which had a preselection bias.

\section{Conclusion}

In this study, we considered that HRQOL maybe decrease the PCHD risk by reducing elevated Lp(a) levels in man. Therefore, we recommend that better HRQOL is a promising protective factor for elevated $L p(a)$ levels in man. Furthermore, more evidences need to be found through multicenter, large- sample, prospective studies to investigate whether better HRQOL can effectively reduce elevated Lp(a) levels and PCHD risk.

\section{Abbreviations}




\begin{tabular}{|c|c|}
\hline Lipoprotein(a) & $\operatorname{Lp}(a)$ \\
\hline Coronary heart disease & $\mathrm{CHD}$ \\
\hline Premature coronary heart disease & PCHD \\
\hline Total Cholesterol & $\mathrm{TC}$ \\
\hline Triglyceride & $\mathrm{TG}$ \\
\hline Low density lipoprotein cholesterol & LDL-C \\
\hline High density lipoprotein cholesterol & HDL-C \\
\hline Apolipoprotein A1 & ApoA1 \\
\hline Apolipoprotein B & ApoB \\
\hline Apolipoprotein E & ApoE \\
\hline Fasting blood glucose & FBG \\
\hline Health related quality of life & HRQOL \\
\hline 36-Item Short Form Health Survey & SF-36 \\
\hline Body mass index & $\mathrm{BMI}$ \\
\hline Physical functioning & $\mathrm{PF}$ \\
\hline Role-physical & $\mathrm{RP}$ \\
\hline Bodily pain & $\mathrm{BP}$ \\
\hline General health & $\mathrm{GH}$ \\
\hline Vitality & VT \\
\hline Social functioning & SF \\
\hline Role-emotional & $\mathrm{RE}$ \\
\hline Mental health & $\mathrm{MH}$ \\
\hline
\end{tabular}

\section{Declarations}

\section{Conflicts of interest}

The authors declare they have no conflicts of interest relevant to this observational retrospective study.

\section{Conflicts of Interest Statement}

The authors declare they have no conflicts of interest relevant to this observational retrospective study.

\section{Acknowledgements}

The author thanks Professor Dengfeng Geng for guiding the project design.

\section{References}

1. Emerging Risk Factors C, Erqou S, Kaptoge S, et al. Lipoprotein(a) concentration and the risk of coronary heart disease, stroke, and nonvascular mortality. Jama. 2009;302:412-23. 
2. Nordestgaard BG, Chapman MJ, Ray K, et al. Lipoprotein(a) as a cardiovascular risk factor: current status. European heart journal. 2010;31:2844-53.

3. Sandkamp M, Funke H, Schulte H, Kohler E, Assmann G. Lipoprotein(a) is an independent risk factor for myocardial infarction at a young age. Clinical chemistry. 1990;36:20-3.

4. Schatz U, Fischer S, Muller G, et al. Cardiovascular risk factors in patients with premature cardiovascular events attending the University of Dresden Lipid Clinic. Atherosclerosis Supplements. 2019;40:94-9.

5. Burgess S, Ference BA, Staley JR, et al. Association of LPA Variants With Risk of Coronary Disease and the Implications for Lipoprotein(a)-Lowering Therapies: A Mendelian Randomization Analysis. JAMA cardiology. 2018;3:619-27.

6. Clarke R, Peden JF, Hopewell JC, et al. Genetic variants associated with Lp(a) lipoprotein level and coronary disease. N Engl J Med. 2009;361:2518-28.

7. Tsimikas S, Fazio S, Ferdinand KC, et al. NHLBI Working Group Recommendations to Reduce Lipoprotein(a)-Mediated Risk of Cardiovascular Disease and Aortic Stenosis. J Am Coll Cardiol. 2018;71:177-92.

8. Anderson TJ, Gregoire J, Pearson GJ, et al. 2016 Canadian Cardiovascular Society Guidelines for the Management of Dyslipidemia for the Prevention of Cardiovascular Disease in the Adult. Can J Cardiol. 2016;32:1263-82.

9. Gencer B, Mach F. Potential of Lipoprotein(a)-Lowering Strategies in Treating Coronary Artery Disease. Drugs. 2020;80:229-39.

10. Khera AV, Everett BM, Caulfield MP, et al. Lipoprotein(a) concentrations, rosuvastatin therapy, and residual vascular risk: an analysis from the JUPITER Trial (Justification for the Use of Statins in Prevention: an Intervention Trial Evaluating Rosuvastatin). Circulation. 2014;129:635-42.

11. Tsimikas S. A Test in Context: Lipoprotein(a): Diagnosis, Prognosis, Controversies, and Emerging Therapies. J Am Coll Cardiol. 2017;69:692-711.

12. Willeit P, Ridker PM, Nestel PJ, et al. Baseline and on-statin treatment lipoprotein(a) levels for prediction of cardiovascular events: individual patient-data meta-analysis of statin outcome trials. Lancet. 2018;392:1311-20.

13. Ray KK, Landmesser U, Leiter LA, et al. Inclisiran in Patients at High Cardiovascular Risk with Elevated LDL Cholesterol. N Engl J Med. 2017;376:1430-40.

14. Cepeda-Valery B, Cheong AP, Lee A, Yan BP. Measuring health related quality of life in coronary heart disease: the importance of feeling well. Int J Cardiol. 2011;149:4-9.

15. Sajobi TT, Wang M, Awosoga O, et al. Trajectories of Health-Related Quality of Life in Coronary Artery Disease. Circulation Cardiovascular quality outcomes. 2018;11:e003661.

16. Graham MM, Norris CM, Galbraith PD, Knudtson ML, Ghali WA, Investigators A. Quality of life after coronary revascularization in the elderly. European heart journal. 2006;27:1690-8.

17. De Smedt D, Clays E, Annemans L, et al. Health related quality of life in coronary patients and its association with their cardiovascular risk profile: results from the EUROASPIRE III survey. Int J Cardiol. 2013;168:898-903.

18. Bonaccio M, Di Castelnuovo A, Costanzo S, et al. Health-related quality of life and risk of composite coronary heart disease and cerebrovascular events in the Moli-sani study cohort. European journal of preventive cardiology. 2018;25:287-97.

19. Ware JE Jr, Sherbourne CD. The MOS 36-item short-form health survey (SF-36). I. Conceptual framework and item selection. Medical care. 1992;30:473-83.

20. Lichtman JH, Froelicher ES, Blumenthal JA, et al. Depression as a risk factor for poor prognosis among patients with acute coronary syndrome: systematic review and recommendations: a scientific statement from the American Heart Association. Circulation. 2014;129:1350-69.

21. Gale CR, Batty GD, Osborn DP, Tynelius P, Rasmussen F. Mental disorders across the adult life course and future coronary heart disease: evidence for general susceptibility. Circulation. 2014;129:186-93.

22. Goff DC Jr, Lloyd-Jones DM, Bennett G, et al. 2013 ACC/AHA guideline on the assessment of cardiovascular risk: a report of the American College of Cardiology/American Heart Association Task Force on Practice Guidelines. Circulation. 2014;129:49-73.

23. Jurges $H$, Avendano M, Mackenbach JP. Are different measures of self-rated health comparable? An assessment in five European countries. Eur J Epidemiol. 2008;23:773-81. 
24. Gencer B, Rigamonti F, Nanchen D, et al. Prognostic value of elevated lipoprotein(a) in patients with acute coronary syndromes. Eur J Clin Invest. 2019;49:e13117.

25. Schwartz GG, Ballantyne CM, Barter PJ, et al. Association of Lipoprotein(a) With Risk of Recurrent Ischemic Events Following Acute Coronary Syndrome: Analysis of the dal-Outcomes Randomized Clinical Trial. JAMA cardiology. 2018;3:164-8.

26. Verweij SL, de Ronde MWJ, Verbeek R, et al. Elevated lipoprotein(a) levels are associated with coronary artery calcium scores in asymptomatic individuals with a family history of premature atherosclerotic cardiovascular disease. J Clin Lipidol. 2018;12:597-603 e1.

27. Kamstrup PR, Benn M, Tybjaerg-Hansen A, Nordestgaard BG. Extreme lipoprotein(a) levels and risk of myocardial infarction in the general population: the Copenhagen City Heart Study. Circulation. 2008;117:176-84.

28. Afshar M, Pilote L, Dufresne L, Engert JC, Thanassoulis G. Lipoprotein(a) Interactions With Low-Density Lipoprotein Cholesterol and Other Cardiovascular Risk Factors in Premature Acute Coronary Syndrome (ACS). Journal of the American Heart Association. 2016; 5.

29. Awad K, Mikhailidis DP, Katsiki N, et al. Effect of Ezetimibe Monotherapy on Plasma Lipoprotein(a) Concentrations in Patients with Primary Hypercholesterolemia: A Systematic Review and Meta-Analysis of Randomized Controlled Trials. Drugs. 2018;78:453-62.

30. Hovingh GK, Kastelein JJ, van Deventer SJ, et al. Cholesterol ester transfer protein inhibition by TA-8995 in patients with mild dyslipidaemia (TULIP): a randomised, double-blind, placebo-controlled phase 2 trial. Lancet. 2015;386:452-60.

31. Group HTRC, Bowman L, Hopewell JC, et al. Effects of Anacetrapib in Patients with Atherosclerotic Vascular Disease. N Engl J Med. 2017;377:1217-27.

32. Kyriakou T, Seedorf U, Goel A, et al. A common LPA null allele associates with lower lipoprotein(a) levels and coronary artery disease risk. Arterioscler Thromb Vasc Biol. 2014;34:2095-9.

33. Whalley B, Rees K, Davies P, et al. Psychological interventions for coronary heart disease. The Cochrane database of systematic reviews. 2011: CD002902.

34. Richards SH, Anderson L, Jenkinson CE, et al. Psychological interventions for coronary heart disease: Cochrane systematic review and meta-analysis. European journal of preventive cardiology. 2018;25:247-59.

35. Catena C, Colussi G, Nait F, Capobianco F, Sechi LA. Plasma lipoprotein(a) levels and atherosclerotic renal artery stenosis in hypertensive patients. Kidney blood pressure research. 2015;40:166-75.

36. Mora S, Lee IM, Buring JE, Ridker PM. Association of physical activity and body mass index with novel and traditional cardiovascular biomarkers in women. Jama. 2006;295:1412-9.

37. Wang W, Lee ET, Alaupovic P, Blackett P, Blevins KS. Correlation between lipoprotein(a) and other risk factors for cardiovascular disease and diabetes in Cherokee Indians: the Cherokee Diabetes Study. Ann Epidemiol. 2005;15:390-7.

38. Katsiki N, Al-Rasadi K, Mikhailidis DP. Lipoprotein (a) and Cardiovascular Risk: The Show Must go on. Curr Med Chem. 2017;24:989-1006.

39. Malmberg K, Bavenholm P, Hamsten A. Clinical and biochemical factors associated with prognosis after myocardial infarction at a young age. J Am Coll Cardiol. 1994;24:592-9.

40. Lippi G, Montagnana M, Luca Salvagno G, Targher G, Cesare Guidi G. Epidemiological association between uric acid concentration in plasma, lipoprotein(a), and the traditional lipid profile. Clinical cardiology. 2010;33:E76-80.

\section{Figures}




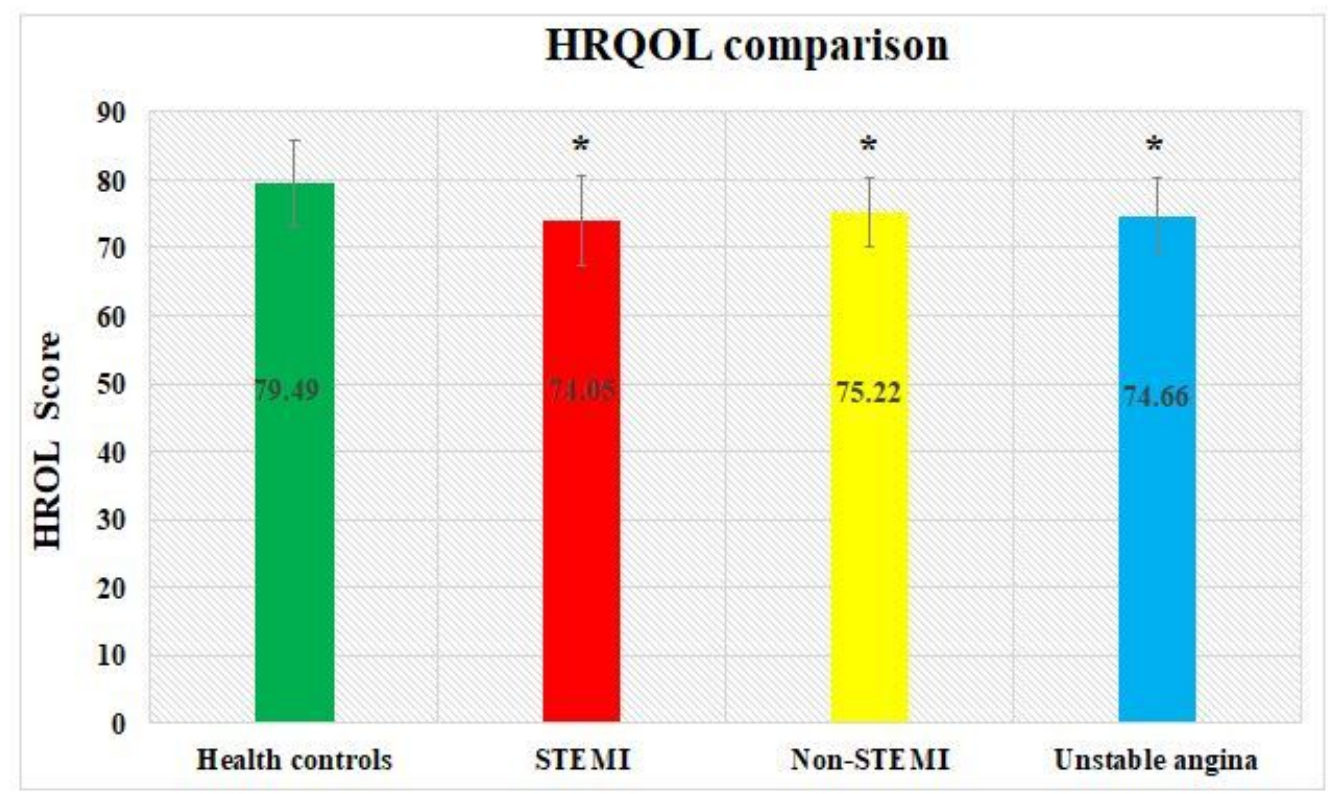

\section{Figure 1}

Correlation with health controls, STMI, Non-STMI and Unstable angina patients were significantly lower HRQOL, but no different in three groups. * Compared with the health controls.

\section{Supplementary Files}

This is a list of supplementary files associated with this preprint. Click to download.

- supplement.docx 